\title{
Indoor Mapping and Positioning Using Impulse Radios
}

\author{
Wenyu Guo, Nick P. Filer \\ School of Computer Science \\ The University of Manchester \\ Oxford Road \\ Manchester, M13 9PL, UK
}

\author{
Rudolf Zetik \\ Electronic Measurement Research Lab \\ Ilmenau University of Technology \\ P.O.Box 100565 \\ 98684 Ilmenau, Germany
}

\begin{abstract}
This paper describes a self-localisation technique suitable for impulse radios operating indoors. Given the times of flight (TOFs) of dominant echoes measured in self-to-self radio channels, we present the algorithms to map typical indoor environments comprising four walls in relation to the radio. This is achieved as follows: first, the type of individual echoes, i.e. a single reflection or a double reflection, and the correspondences among them are identified; based on these, substructures of the surrounding environment can be reconstructed; by combining these substructures, a complete 2D map outlining the environment can be derived. When there is more than one radio, mapping information in individual radios can be exchanged and combined to show a more complete representation of the surrounding environment. The technique can be extended to solve more complex scenarios.
\end{abstract}

Index Terms-Radiolocation, Impulse radio, UWB, Mapping

\section{INTRODUCTION}

The Global Positioning System (GPS), the dominant system offering location information outdoors, suffers a poor indoor performance due to low signal availability, as GPS signals are not designed to penetrate through most construction materials. This has recently made indoor localisation a hot research topic. Many indoor positioning techniques have been developed, most of which rely on fixed references to determine the location of tagged devices [1-5]. This architecture implies that location information is only available in environments where references with known positions have been deployed. However, in many scenarios where location information is very useful, a reference system is not likely to have been previously deployed. For example, when a fire brigade is rescuing people from a smoke-filled building, a fireman needs to know his location in order to find the nearest exit or corridor. Also, a fireman needs to know the position of his colleagues in order to help or get help from them. In this case, there is no time for the fire brigade to deploy a reference system before entering the building. Therefore, an indoor wireless system providing location information without fixed references would be useful.

In situations where a reference system is not available, individual mobile nodes need to locate themselves. Therefore, we have been investigating indoor location sensing using individual radio-equipped mobile nodes. This paper describes a technique suitable for an impulse radio to map the surrounding environment and pinpoint itself in relation to the environment.

This technique uses transmitters and their images in the walls as references. These images are spontaneously synchronised with the transmitters, i.e. whenever the transmitter transmits a pulse, all its images "transmit" at the same time. This solves the problem of synchronisation among references, which is usually very costly to implement in conventional positioning systems, e.g. the Global Positioning System equips individual satellites with atomic clocks for synchronisation purposes.

Reducing the complexity of the system is one of the most efficient methods to reduce the cost. To make the system simple, receivers are insensitive to amplitude or phase. Only times of flight (TOFs) of echoes with energy above the receiving threshold can be detected in individual radios. It has been found in indoor multipath measurements at a range of frequencies [6,7] that received echoes comprise a small number of dominant echoes from large flat surfaces like walls, interspersed with noise-like scattering from smaller objects across the entire range of delays. We found the same phenomenon from indoor channel measurements at Ultra Wideband (UWB) bandwidth, which ranges from $3.1 \mathrm{GHz}$ to $10.6 \mathrm{GHz}$, according to FCC rules part 15.517(b) [8]. Some indoor channel measurements and results are discussed in section III of this paper.

It can be expected that by suitable thresholding on receivers, only dominant echoes including the direct line-ofsight (LOS), single reflections and most double reflections are detected. TOFs of these dominant echoes can then be used to map the major features of the environment. This has similarities to image ray-tracing [9].

The calculations are not trivial, but they only need to be updated at a sufficient rate to update the mapping information and track the movements of radio users. This is expected to be within the capacity of impulse radios and therefore can be done by individual users locally. The timer in the receiver of an impulse radios can resolve down to near 10ps time delay [10], which means approximately $0.3 \mathrm{~cm}$ path length accuracy. Based on this, the accuracy of the proposed technique is expected to be within a few centimetres in practice, taking into account other errors introduced by pulse distortions, geometric dilution of position (GDOP) [11] etc. If the 
transmitter and the receiver in the radio are sharing the same antenna, there may be some minimum delay after the transmitter transmits the pulse and before the receiver fully recovers its sensitivity. This gives a blind area close to the radio. Though a radio user may not be able to detect objects within this blind area, it usually detects them before they enter this area. Other techniques such as infrared or sonar can be used for object detection in the blind area. Therefore, in general it will not affect the result of environment reconstruction.

This paper uses 2D scenarios with a single radio user operating in various closed four-wall environments to show how this technique can be used to map these scenarios and even more complex ones.

\section{THE STORY OF THIS RESEARCH}

This research was originally started from mapping very simple 2D indoor environments using the times of arrival of dominant echoes measured by two impulse radios, as two radios can be treated as a subset of a radio network. Algorithms for two radios to reconstruct a single wall in range and pinpoint themselves in relation to the wall were developed first. Basically, either radio can position the other one on a hyperbola by measuring the difference between the TOFs of the LOS signal and the single reflection from the other radio, where the foci of the hyperbola are the radio itself and its image in the wall.

Algorithms for two radios to reconstruct various environments comprising two walls forming an acute, right or obtuse angle were developed and are described in [12,13]. We then developed 2D algorithms to reconstruct typical indoor geometries such as four-wall rooms, which are described in [14], followed by a feasibility study of using two radios to reconstruct $2.5 \mathrm{D}$ indoor environments with walls perpendicular to the floor and ceiling [15].

By reconstructing more and more complex environments, we found that as the number of walls in the environment increases, the amount of information for environment reconstruction increases faster than the complexity introduced by additional walls, though it takes more computation power to reconstruct an environment comprising more walls. For this reason, we carried out a feasibility study to see whether it is possible to reconstruct typical indoor environments such as a four-wall room using a single radio. Using a single impulse radio to reconstruct a closed four-wall geometry is discussed in sections IV of this paper.

\section{INDOOR CHANNEL MEASUREMENTS}

Measurements have been carried out in order to find out whether, in UWB bandwidth, received echoes comprise a small number of dominant echoes from large flat surfaces like walls, interspersed with noise-like scattering from smaller objects. The other purpose of the measurements is to prove the assumption that, by suitable thresholding on receivers, dominant pulses including the LOS, single reflections and most double reflections can be filtered out from non-dominant ones. We are also attempting to reconstruct scenarios from measured data and to compare the results with software simulations.

\section{A. The measurement equipment}

The measurements were carried out using a channel sounder, which adopts binary sequences to stimulate a radio channel. Fig. 1 shows the fundamental architecture of the channel sounder at base-band. Its detailed description and extensions into higher frequency bands can be found in [16]. Fig. 2 shows a photo of the multi-channel UWB channel sounder built in a 19-inch rack, which is designed at Ilmenau University of Technology in cooperation with MEODAT [17] in the framework of the EU project PULSERS [18].

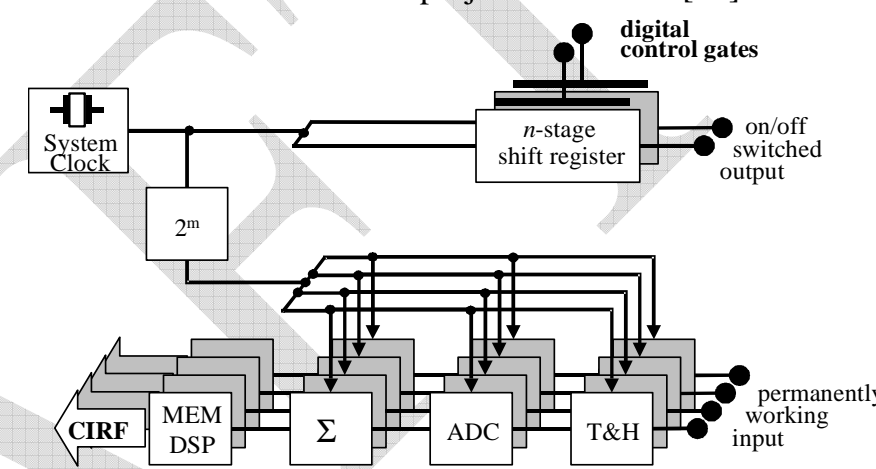

Fig. 1. Architecture of the real-time UWB MIMO channel sounder

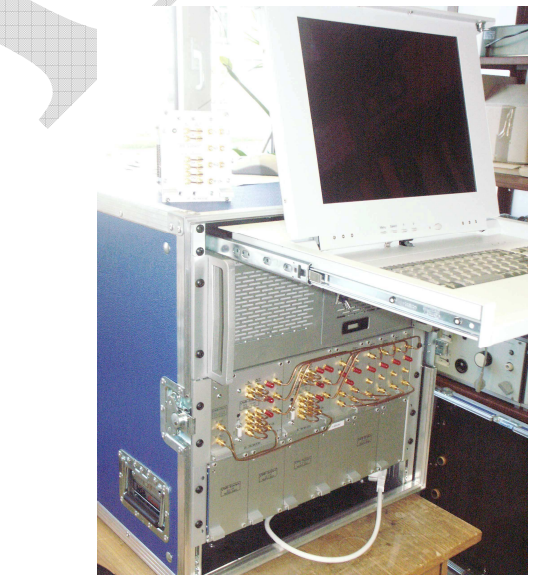

Fig. 2. Real-time UWB MIMO channel sounder

The shift-register, binary divider and $\mathrm{T} \& \mathrm{H}$ of the sounder are implemented using SiGe monolithic integrated circuits. In contrast with conventional sequential sampling oscilloscopes, synchronous digital controlled sub-sampling adopted by the sounder offers extremely linear time with superior jitter and drift behaviour. The DSP module is based on standard offshelf PCB products. The sounder is driven by a $7 \mathrm{GHz}$ system clock, which gives a bandwidth of $3.5 \mathrm{GHz}$. It operates in the base-band (DC-3.5GHz) as well as in frequency band from $3.5 \mathrm{GHz}$ to $10.5 \mathrm{GHz}$ and its extension to $60 \mathrm{GHz} \pm 3.5 \mathrm{GHz}$ has already been tested in the laboratory. The sounder generates $585 \mathrm{~ns}$ M-sequences with a repetition rate up to 3300 sequences per second. It offers dynamic range over $55 \mathrm{~dB}$. The sounder has 2 transmitters and 4 receivers. 


\section{B. Indoor channel measurement setup}

The measurement was carried out in an office environment with the channel sounder operating at the frequency band from $3.5 \mathrm{GHz}$ to $10.5 \mathrm{GHz}$. The floor plan of the measurement environment is shown in Fig. 3. A mobile transmitting antenna (Tx) and a fixed receiving antenna $(\mathrm{Rx})$ were used. These antennas were $1.81 \mathrm{~m}$ above the floor. Their positions were measured using a laser. The transmitting antenna was moved by a person during the measurements. In this experiment, the transmitting antenna approaches the four surrounding walls individually, as shown in Fig. 4, to make it easier to identify dominant echoes from individual walls using the measured data.

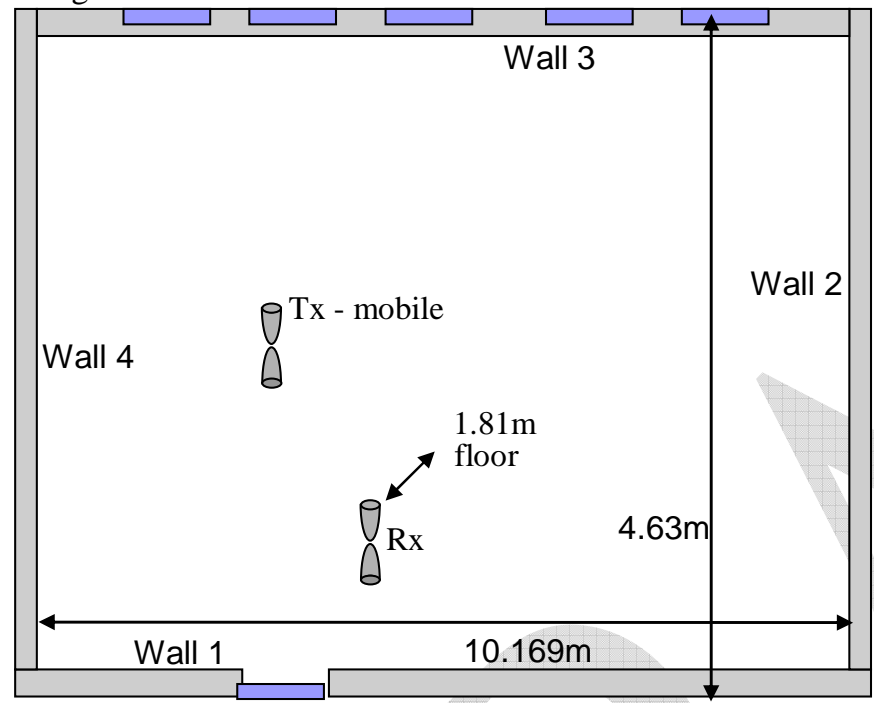

Fig. 3. The measurement scenario

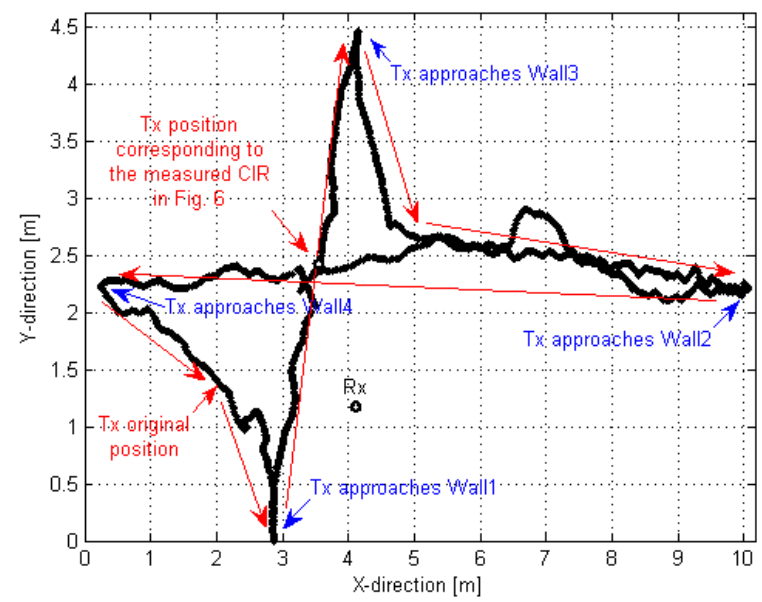

Fig. 4. Movement track of the transmitting antenna Tx

\section{The results}

Channel impulse responses (CIRs) measured by $\mathrm{Rx}$ are shown in Fig. 5. These comprise 1899 samples taken with a constant time interval. The vertical axis represents the TOFs of received impulses in relation to the time of transmission, while the horizontal axis shows the index of the measurement samples. Thus, in Fig. 5, each vertical line represents a measured CIR. An example of a measured CIR is shown in
Fig. 6.

As the transmitting antenna $\mathrm{Tx}$ approaches a wall, the TOFs of the LOS signal and the single reflection from the wall received by the receivers becomes similar. Knowing this, we can easily identify the correspondences between dominant echoes in a measured CIR and the surrounding walls, e.g. the single reflections from Wall 1, 2, 3 and 4 are indicated individually in Fig. 6. Fig. 6 also shows a sample thresholding, by which dominant pulses including the LOS, single reflections and most double reflections can be selected out from non-dominant ones. The method to find a suitable threshold is described in [19].

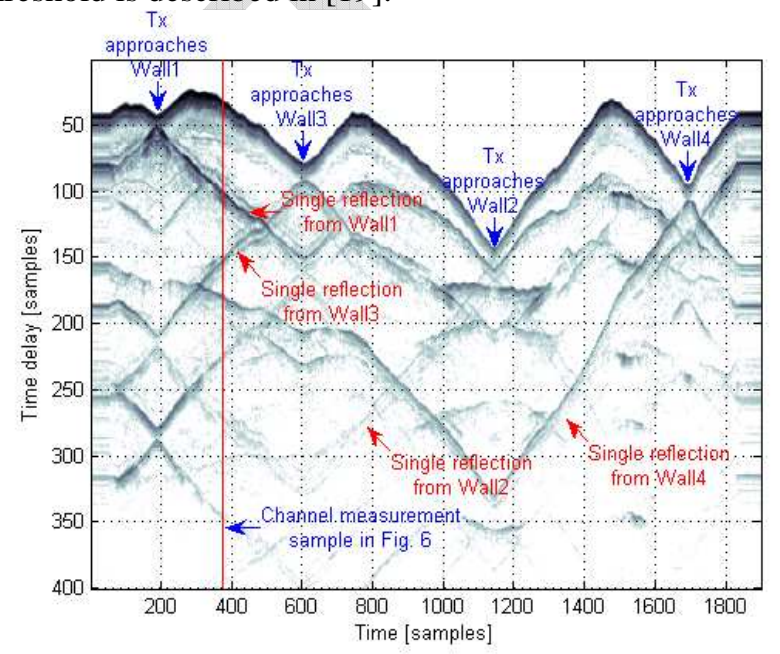

Fig. 5. Data measured by receiving antenna $\mathrm{Rx}$

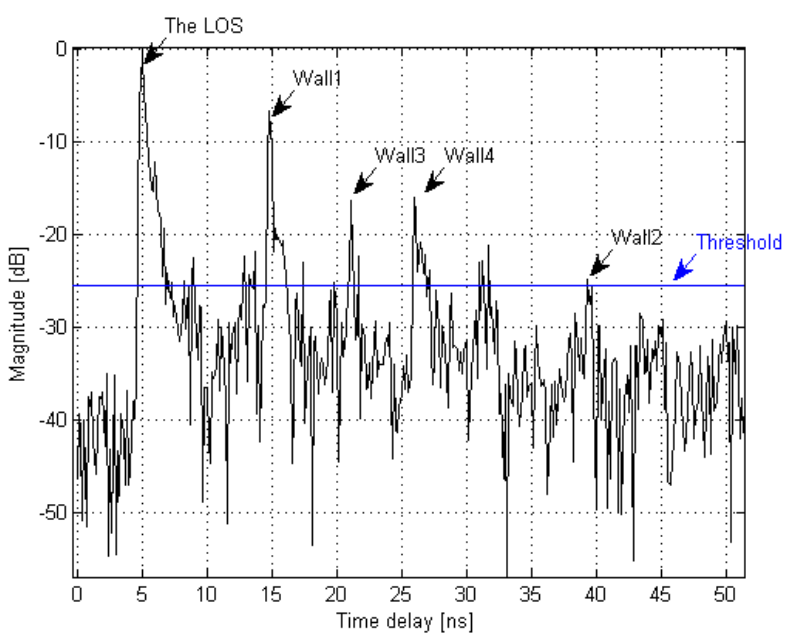

Fig. 6. A measured channel impulse response

\section{ClOSED FOUR-WALl ENVIRONMENT RECONSTRUCTION} USING A SINGLE IMPULSE RADIO

Being able to select the dominant echoes in receivers by suitable thresholding, we carried out a feasibility study on using a single impulse radio to reconstruct closed four-wall environments, which type of geometries are the most common indoors.

For a closed four-wall environment, the total value of the four inner angles is $2 \pi$. As, in reality, corners of typical four- 
wall indoor environments are either slightly greater or less than $\pi / 2$ instead of exactly square, there are three possible combinations of the four inner angles: one obtuse and three acute; two obtuse and two acute; three obtuse and one acute. As the total value of four acute angles is less than $2 \pi$ and the total value of four obtuse angles is greater than $2 \pi$, they are not possible combinations.

When a radio user measures TOFs, because dominant echoes usually consist of single reflections and some double reflections, it is assumed that, by suitable thresholding, the radio detects these echoes only, and ignores most or all higher-order reflections. Roundtrip delays from time of transmission to times of arrival of dominant echoes are recorded as a delay pattern, which, for the measured environment, uniquely corresponds to the scenario at the time of transmission.

When a single radio is transmitting and receiving in a fourwall environment, any two walls forming an acute angle can contribute a double reflection. In most closed four-wall indoor environments, a wall is either roughly perpendicular or parallel to the others. Therefore, a double reflection can be either from two roughly perpendicular walls forming an angle slightly smaller than $\pi / 2$, or two roughly parallel walls.

When there is a double reflection around the corner of two roughly perpendicular walls, the two reflection points will be very close to each other. Therefore, these two walls are adjacent. This type of double reflection is called an 'aroundthe-corner' double reflection. The number of around-thecorner double reflections should be the same as the number of inner acute angles. When a double reflection is reflected by two roughly parallel walls, these two walls should not be adjacent, since even if there is an intersection, it will be out of the detection range of the radio. This type of double reflection is called a 'bouncing' double reflection. As most closed fourwall indoor geometries can be divided into two pairs of roughly parallel walls, there are usually two bouncing double reflections.

Therefore, the delay pattern corresponding to an environment with one obtuse and three acute inner angles usually consists of the TOFs of four single reflections, each from a wall, three around-the-corner double reflections and two bouncing double reflections, which is nine in total. The delay pattern corresponding to an environment with two obtuse and two acute inner angles usually consists of eight TOFs, as there are only two around-the-corner double reflections. For the same reason, the delay pattern corresponding to an environment with three obtuse and one acute inner angle usually consists of seven TOFs.

Environment mapping as discussed in this paper uses measured delay patterns consisting of TOFs of echoes to position surrounding walls relative to the radio user. We will first investigate scenarios with one obtuse and three acute angles, where nine TOFs are available. Mapping of this class of scenarios, which is discussed in section IV.A of this paper, is expected to be simpler than mapping others with the same number of walls but fewer TOFs available. Mapping of scenarios with two obtuse and two acute inner angles will be discussed next in sections IV.B and IV.C, where the two obtuse angles can be adjacent or opposite to each other. Mapping of scenarios with one acute and three obtuse inner angles will be discussed in section IV.D.

\section{A. Scenarios With One Obtuse and Three Acute Inner Angles}

1) The scenario and the delay pattern

Fig. 7 shows a single radio user (R) operating in a closed four-wall environment with three acute and one obtuse inner angle. The delay pattern measured by the user is shown in Fig. 8, which consists of the TOF of nine echoes. Initially, the radio user has no knowledge of the surrounding environment.

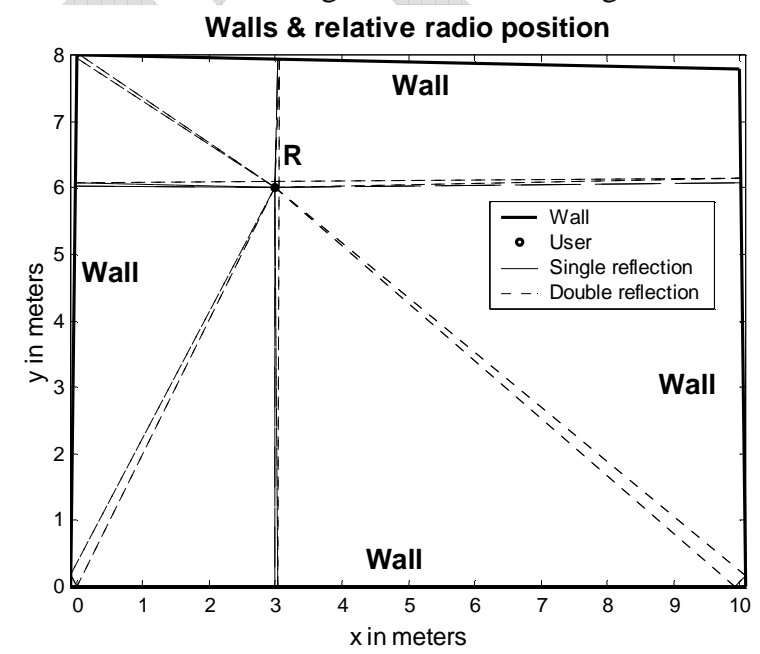

Fig. 7. A single radio operating in a four-wall environment with three acute inner angles

Measured Delay Pattern R-->R

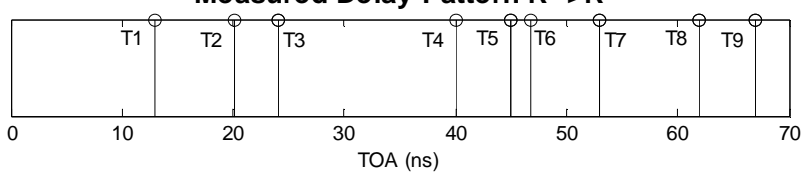

Fig. 8. Delay pattern corresponding to the scenario shown in Fig. 7

As three surrounding walls can contribute at most six echoes corresponding to a single pulse transmitted by the user, while four walls can contribute up to nine, there must be at least four walls in the environment, assuming any triple reflections are below the threshold.

Using TS to represent the TOF of a single reflection and TD to represent the TOF of a double reflection, the TOF of a double reflection reflected by walls $\mathrm{A}$ and $\mathrm{B}$ (TDab) will be related to the TOFs of single reflections from WallA (TSa) and WallB (TSb). This relation can be used to identify double reflections.

\section{2) Partial map reconstruction}

When WallA and WallB are perpendicular to each other, $T D a b=\sqrt{T S a^{2}+T S b^{2}}$. 
$T D a b=T S a+T S b$.

As two walls contribute a double reflection only when they form an angle between 0 and $\pi / 2$,

$$
\sqrt{T S a^{2}+T S b^{2}} \leq T D a b \leq T S a+T S b .
$$

The exact value of an acute or right angle $\alpha_{a b}$ between walls can be calculated using

$$
\alpha_{a b}=\arccos \frac{T D a b^{2}-T S a^{2}-T S b^{2}}{2 \times T S a \times T S b} .
$$

Assume we know that a particular TD (TDab) corresponds to two TSs (TSa and TSb). The angle between the normals from the user (R) to the two walls (WallA and WallB) can be calculated as $\pi-\alpha_{a b}$. The distance from the user to either wall can be calculated using

$$
d=T S \times c / 2 \text {, }
$$

where $\mathrm{c}$ is the speed of light. Based on this, possible positions of walls can be derived in relation to the radio. By defining a Cartesian coordinate system centred at the user, with the path of a single reflection represented by either TS lying on the negative vertical axis, e.g. the single reflection from WallA, two possible partial maps consisting of the two walls and the user can be derived as shown in Fig. 9. These two maps are mirror images of each other.

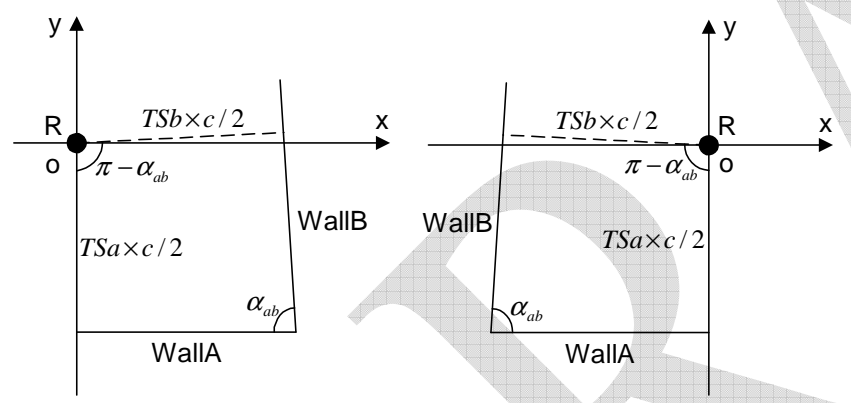

Fig. 9 Possible partial maps reconstructed based on the knowledge of a TD and its corresponding TSs

In most typical indoor environments, a wall, e.g. WallA, forms an acute angle with a set of more than one other wall. As any wall from the set can be positioned in relation to WallA and the user, a more complete map of the scenario can be derived by positioning all walls from the set in relation to WallA and the user. As individual walls may form acute angles with a set of different numbers of other walls, one of them should corresponds to the largest set consisting of most or all other surrounding walls. This wall is called 'the reference wall'. A possible map reconstructed based on 'the reference wall' will be the most complete representation of the scenario. This map can be presented in a Cartesian coordinate system centred at the user with the normal to 'the reference wall' lying on the negative vertical axis.

\section{3) Accuracy}

Equation (4) suggests the accuracy of reconstructed twowall partial maps is related with the curve of arc cosine, as shown in Fig. 10. From the figure, it can be found that $\arccos (x)$ becomes more and more sensitive to the value of $\mathrm{x}$ as it decreases from 1 to 0 . This is because the derivative of $\arccos (x),-\frac{1}{\sqrt{1-x^{2}}}$, decreases as $\mathrm{x}$ increases. According to (4), we conclude that the best accuracy is achieved when the angle $\alpha_{a b}$ is close to $\pi / 2$, as it is the most sensitive to the value of $\frac{T D a b^{2}-T S a^{2}-T S b^{2}}{2 \times T S a \times T S b}$. On the other hand, if the calculated value of $\alpha_{a b}$ is close to zero, it is less accurate.

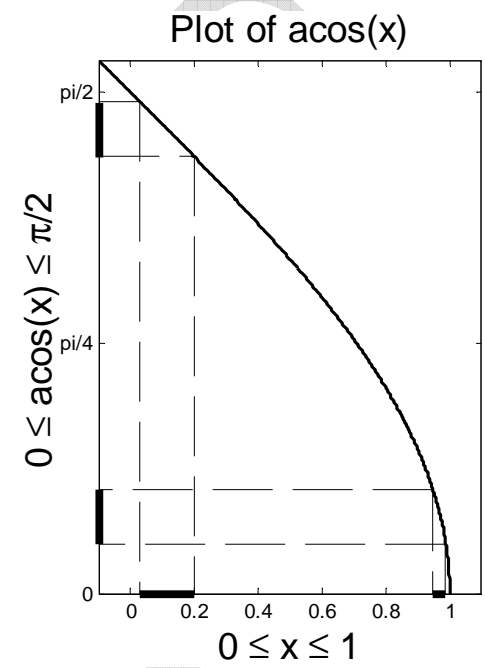

Fig. 10 The curve of arc cosine

4) Correspondence between double and single reflections

Before reconstructing either possible partial or complete maps, the radio user needs to identify whether a received echo is a single reflection or a double reflection and the correspondence between double and single reflections. As individual walls are either roughly perpendicular or parallel to the others, the most likely value of a TD (TDab) corresponding to two single reflections (TSa and TSb) will fall into two very small ranges. When the two reflecting walls are roughly perpendicular to each other, TDab can be equal to or a little greater than $\sqrt{T S a^{2}+T S b^{2}}$, i.e.

$T D a b \approx \sqrt{T S a^{2}+T S b^{2}} \cap T D a b>\sqrt{T S a^{2}+T S b^{2}}$.

Alternatively, when the two reflecting walls are roughly parallel to each other, TDab can be equal to or a little smaller than $T S a+T S b$, i.e.

$T D a b \approx T S a+T S b \bigcap T D a b<T S a+T S b$.

If the value of a TOF is smaller than $\sqrt{T S a^{2}+T S b^{2}}$ or greater than $T S a+T S b$, it cannot represent a TD corresponding to $\mathrm{TSa}$ and $\mathrm{TSb}$.

\section{5) Correspondence ambiguity}

In reality, the maximum difference in angle from $\pi / 2$ to an angle formed by two roughly perpendicular walls should usually be the same as the maximum difference from zero to an angle formed by two roughly parallel walls. Allowing the same angular difference in geometry, when (6) is satisfied, in order to accommodate a much higher sensitivity, the 
maximum possible value of $T D a b-\sqrt{T S a^{2}+T S b^{2}}$ will be considerably larger than the maximum possible value of $(T S a+T S b)-T D a b$, while (7) is satisfied. This suggests when (6) is satisfied, apart from Tab, it is very likely to find other TDs, e.g. TDcd, to satisfy the relation of

$\left|T D c d-\sqrt{T S a^{2}+T S b^{2}}\right|<\left|T D a b-\sqrt{T S a^{2}+T S b^{2}}\right| \max$.

TDcd therefore could be the TD corresponding to TSa and TSb. This ambiguity is called "correspondence ambiguity". However, when (7) is satisfied, as the maximum possible value of $(T S a+T S b)-T D a b$ is small, apart from TDab, it is very unlikely to find any other TD, e.g. TDcd, to satisfy the relation of

$(T S a+T S b)-T D c d<((T S a+T S b)-T D a b)_{\max }$.

Based on this, we designed a procedure to identify the correspondences among TOFs of double and single reflections in a delay pattern, in order to effectively avoid the occurrence of correspondence ambiguity: the correspondences among TOFs of single and double reflections derived from roughly parallel walls should be searched first, as correspondence ambiguity is very unlikely to occur in this searching; once the bouncing double reflections derived from roughly parallel walls are identified, they will be "removed" from the delay pattern, which results in a sparser pattern; the correspondences among TOFs derived from roughly perpendicular walls will then be searched in this sparser pattern, which gives a lower chance for correspondence ambiguities to occur.

6) Identifying the correspondence among echoes

Based on this, the radio user can start mapping by identifying whether a received echo is a single reflection or double reflection and finding the correspondence between double and single reflections.

The nine measured TOFs in Fig. 8 are numbered as T1 to T9 sequentially. As each wall contributes one and only one single reflection, there is a correspondence between each TS and a wall. In the following discussion, a wall will be numbered based on the number of its corresponding TS, e.g. if T6 is found to be a TS, the wall corresponding to T6 is numbered as Wall6.

Among the nine TOFs, the shorter the TOF, the more likely for it to represent a single reflection. T1 and T2 should be TSs, as any double reflection should arrive later than the two single reflections corresponding to it. As discussed, echoes from roughly parallel walls are searched first, beginning with TOFs close to $\mathrm{T} 1+\mathrm{T} 2$, then $\mathrm{T} 1+\mathrm{T} 3, \mathrm{~T} 2+\mathrm{T} 3, \mathrm{~T} 1+\mathrm{T} 4$, etc. It is found that

$T 7 \approx T 1+T 4$

$T 9 \approx T 2+T 6$.

Based on this, T4 and T6 are found to be the other two TSs beside $\mathrm{T} 1$ and $\mathrm{T} 2$. $\mathrm{T} 7$ and $\mathrm{T} 9$ are identified as TDs representing bouncing double reflections. Therefore, the remaining TOFs, T3, T5 and $\mathrm{T} 8$ are expected to be TDs representing around-the-corner double reflections. By searching for echoes from roughly perpendicular walls, beginning with TOFs close to $\sqrt{T 1^{2}+T 2^{2}}$, then $\sqrt{T 2^{2}+T 4^{2}}$, $\sqrt{T 1^{2}+T 6^{2}}$ and $\sqrt{T 4^{2}+T 6^{2}} \cdot \sqrt{T 1^{2}+T 4^{2}}$ and $\sqrt{T 2^{2}+T 6^{2}}$ are skipped, as the relations in (10) and (11) have been found. As expected for our example, it is found that

$$
\begin{aligned}
T 3 & \approx \sqrt{T 1^{2}+T 2^{2}} \\
T 5 & \approx \sqrt{T 2^{2}+T 4^{2}} \\
T 8 & \approx \sqrt{T 4^{2}+T 6^{2}} .
\end{aligned}
$$

The relations in (10-14) suggest that Wall2 is roughly perpendicular and adjacent to Wall1 and Wall4; Wall4 is roughly perpendicular and adjacent to Wall2 and Wall6; Wall2 is roughly parallel to Wall6; Wall1 is roughly parallel to Wall4. The exact angle between walls can be calculated using (4). The distances from walls to the radio can be calculated using (5). The whole scenario can then be reconstructed in a coordinate system defined using the radio and a wall whose position is known in relation to the radio and the other three walls, i.e. Wall 2 or Wall4. Using Wall4 and the radio to define the coordinate system, two possible scenarios, which are mirror images of each other, are reconstructed as shown in Fig. 11.

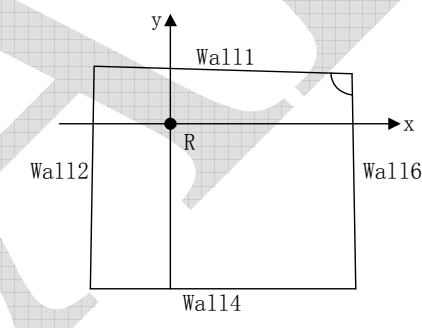

(a)

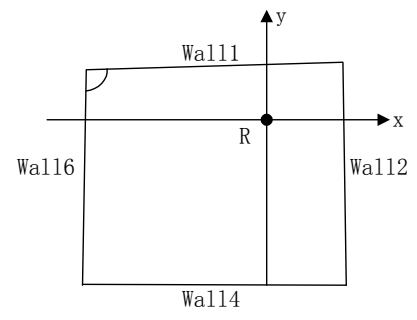

(b)
Fig. 11 Reconstructed possible scenarios in the coordinate system defined by Wall4 and the radio

It can be found from the reconstruction that the geometry of the environment can be determined using only three out of the five relations of (10-14). This raises a selectivity issue among them. Relations providing higher positioning accuracy should be used. In order to achieve the best accuracy, (12-14) which suggest roughly right angles between walls are chosen.

Comparing the two scenarios in Fig. 11 with the one in Fig. 7, it can be found that scenario (a) in Fig. 11 is the correct one, while (b) is its mirror image. This mirror-image ambiguity can be resolved if the user is detected to have moved twice in different directions. This ambiguity will be discussed in section III of this paper.

\section{B. Scenarios With Two Adjacent Obtuse Inner Angles}

Fig. 12 shows a single radio user (R) operating in a closed four-wall environment with two adjacent obtuse inner angles. For a clearer explanation, acute and obtuse inner angles in this scenario are exaggerated. The measured delay pattern consists of eight TOFs, numbered from $\mathrm{T} 1$ to $\mathrm{T} 8$ sequentially, as shown in Fig. 13. 


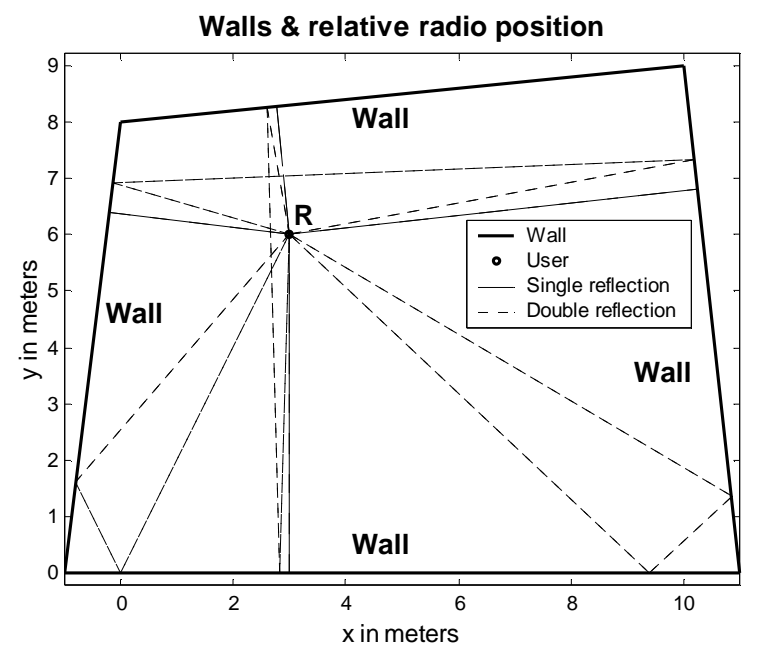

Fig. 12 A single radio (R) operating in a four-wall environment with two adjacent obtuse inner angles

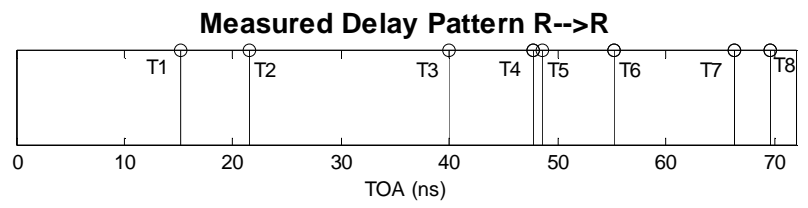

Fig. 13 Delay pattern corresponding to the scenario shown in Fig. 12

By using the same method as described in section II.A, the following correspondences between TSs and TDs are found:

$T 8 \approx T 2+T 5$.

$T 6 \approx T 1+T 3$.

$T 4 \approx \sqrt{T 2^{2}+T 3^{2}}$

$T 7 \approx \sqrt{T 3^{2}+T 5^{2}}$

The relations in (15-18) suggests: $\mathrm{T} 1, \mathrm{~T} 2, \mathrm{~T} 3$ and $\mathrm{T} 5$ are TSs; T6 and T8 are TDs representing bouncing double reflections; $\mathrm{T} 4$ and $\mathrm{T} 7$ are $\mathrm{TDs}$ representing around-thecorner double reflections. Therefore, Wall2 and Wall5 are roughly parallel. Wall3 is adjacent and roughly perpendicular to Wall2 and Wall5, and it is parallel to Wall1. Based on this, as Wall3 can be positioned in relation to all the other three walls and the radio user, it is used to define the coordinate system together with the radio.

Again, there is a selectivity issue among the four relations of (15-18), three of them will be chosen in order to achieve the best accuracy. Relations (17) and (18) are chosen, as they suggest roughly right angles between walls, which are of high accuracy. One of (15) and (16), which suggests low accuracy, must be chosen. As the greater the angle between walls suggests the higher accuracy, the one in (15) and (16) suggesting a greater angle between walls will be used together with (17) and (18) to optimise the reconstruction. However, as a relation of low accuracy is used, the optimised scenario is still of low accuracy.

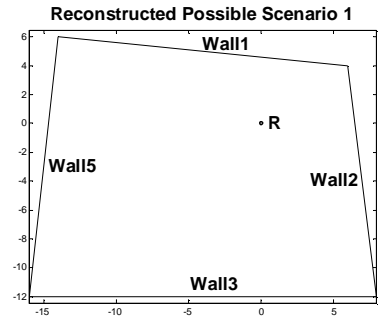

(a)

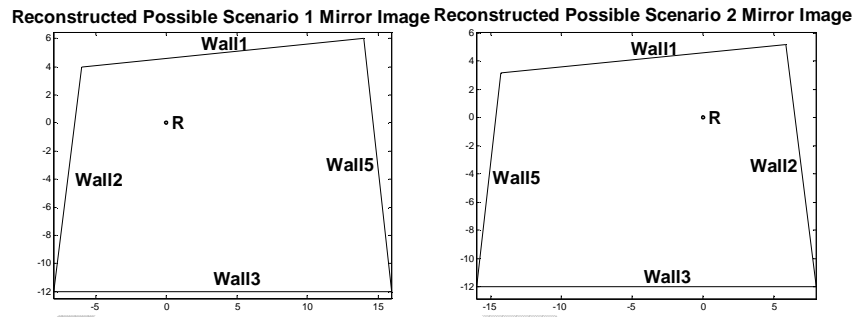

(c)

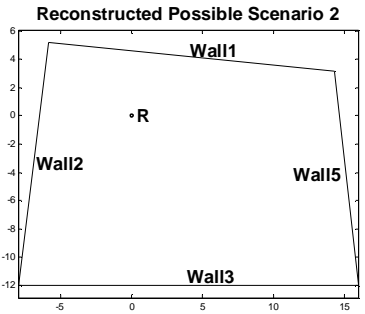

(b)

(d)
Four possible scenarios are reconstructed as shown in Fig. 14. All of them fit the measured delay patterns. Comparing the reconstructed possible scenarios in Fig. 14 with the scenario in Fig. 12, scenario (c) in Fig. 14 is found to be the correct one, while (a) is its mirror image. The other two scenarios (b) and (d) are also mirror images of one another. By comparing (b) and (c), it can be found that the angle between Wall1 and Wall3 is minus the correct angle, while other walls and the radio locate at the same positions. This arises because arc cosine has even symmetry. Both walls adjacent to Wall1 form an obtuse angle with it. As walls forming an obtuse angle do not contribute a double reflection, the radio knows only these two walls form an obtuse angle. If a wall has an obtuse angle with both adjacent walls, the scenario with this wall rotated by double its angle from the roughly parallel wall may also fit the measured delay pattern, provided the rotated wall still forms an obtuse angle with both adjacent walls. For a simpler explanation, in the following discussion, this kind of wall, which can be rotated in the scenario, is called a 'flippable wall'. This ambiguity is called 'flipping-wall ambiguity'.

In order to be 'flippable', a wall needs to have obtuse angles with both adjacent walls both before and after the flip. This is explained in detail as follows.

The scenario shown in Fig. 15 describes the same scenario shown in Fig. 12. Fig. 16 shows the scenario derived by flipping Wall1 in Fig. 15 through the vertical axis. For simpler explanations, in the rest of the paper, the angle from WallA to WallB is named as $\alpha_{a b}$, e.g. the angle from Wall1 to Wall3 is named as $\alpha_{13}$. The angle from AxisX to WallA is named as $\alpha_{x a}$, e.g. the angle from the vertical axis to Wall2 is named as $\alpha_{y 2}$. 


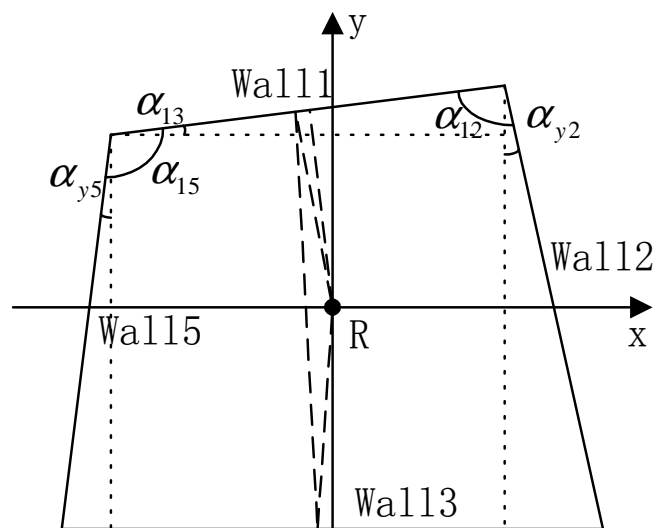

Fig. 15. Four-wall environment with two adjacent obtuse inner angles

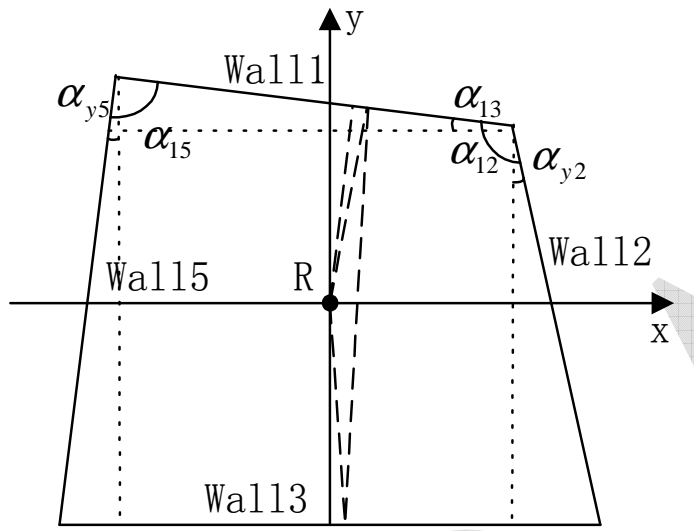

Fig. 16. Scenario derived by flipping Wall1 through the vertical axis

In Fig. 15, both $\alpha_{12}$ and $\alpha_{15}$ are obtuse angles, which suggests no double reflections around either corner. Therefore, Wall1 contributes a single reflection and a double reflection in total, whose reflection paths are represented by dashed lines. By flipping Wall1 through the vertical axis, the reflection path of the corresponding single reflection and double reflection will also flip through the vertical axis, as shown in Fig. 16. If both $\alpha_{12}$ and $\alpha_{15}$ in Fig. 16 are still obtuse, which suggests no double reflection around either corner, the delay patterns corresponding to the scenarios in Fig. 15 and Fig. 16 will be the same. Based on this, for Wall1 to be 'flippable', $\alpha_{12}$ and $\alpha_{15}$ in both Fig. 15 and Fig. 16 need to be obtuse, i.e. in Fig. 15

$\alpha_{12}=\pi / 2+\alpha_{y 2}-\alpha_{13}>\pi / 2$

$\alpha_{15}=\pi / 2+\alpha_{y 5}+\alpha_{13}>\pi / 2$

and in Fig. 16

$\alpha_{12}=\pi / 2+\alpha_{y 2}+\alpha_{13}>\pi / 2$

$\alpha_{15}=\pi / 2+\alpha_{y 5}-\alpha_{13}>\pi / 2$.

In Fig. 15, (19) suggests

$\alpha_{y 2}>\alpha_{13}$.

In Fig. 16, (22) suggests $\alpha_{y 5}>\alpha_{13}$.

As $\alpha_{12}, \alpha_{y 2}$ and $\alpha_{y 5}$ in both Fig. 15 and Fig. 16 are of the same values, the relations in (23) and (24) are satisfied in both figures and these are the basic requirements for Wall1 to be 'flippable'. As in common indoor geometries $\alpha_{y 2}$ and $\alpha_{y 5}$ are usually small, $\alpha_{13}$ is close to zero. Therefore, generally as the angles between roughly parallel walls are close to zero, the errors introduced by the flipping-wall ambiguity are usually small.

\section{Scenarios With Two Opposite Obtuse Inner Angles}

Fig. 17 shows a single radio user (R) operating in a closed four-wall environment with two opposite obtuse inner angles. The delay pattern measured by the user consists of eight TOFs.

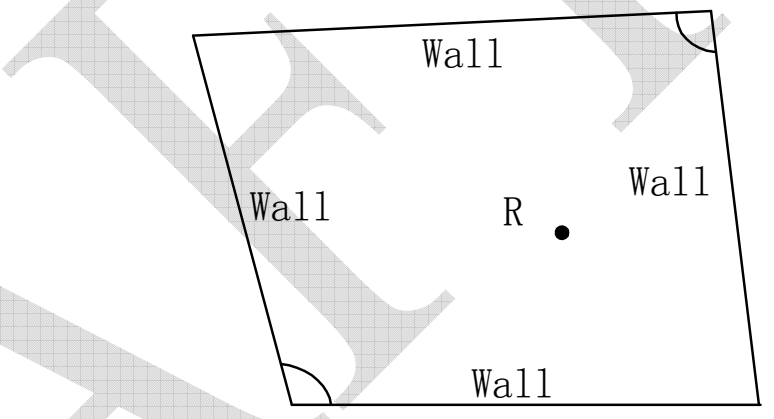

Fig. 17. A single radio (R) operating in a four-wall environment with two opposite obtuse inner angles

By using the same method as described in section II.A, numbering walls according to the rank order of their corresponding TSs, it is found that: Wall1 and Wall5 are roughly parallel; Wall2 and Wall3 are roughly parallel; Wall1 and Wall2 are roughly perpendicular and adjacent; Wall3 and Wall5 are roughly perpendicular and adjacent.

As each of the four walls forms an acute angle with two other walls, as discussed, any wall can be used to define the coordinate system. Using Wall2 to set up the coordinate system centred at the radio user with the path of the single reflection from Wall2 lying on the negative vertical axis, putting aside mirror-image ambiguity, Fig. 18 shows the relative position of Wall1, which is roughly perpendicular and adjacent to Wall2.

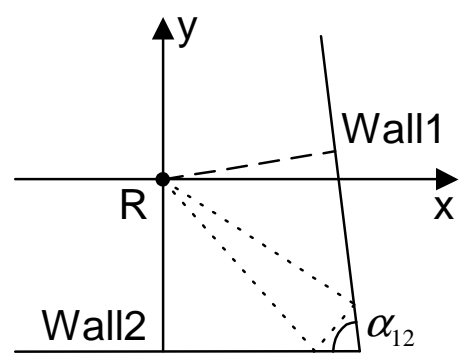

Fig. 18. Position of Wall1 in relation to Wall2 and the radio user ignoring mirror-image ambiguity

By introducing the other pair of roughly perpendicular 
walls forming an acute angle, Wall3 and Wall5, into this map, a complete map of the whole scenario can be reconstructed. The relative position between these two pairs of walls can be determined by checking the angle between roughly parallel walls: $\alpha_{15} \& \alpha_{23}$, which can be calculated using (4). Fig. 19 shows a reconstructed map of the whole scenario.

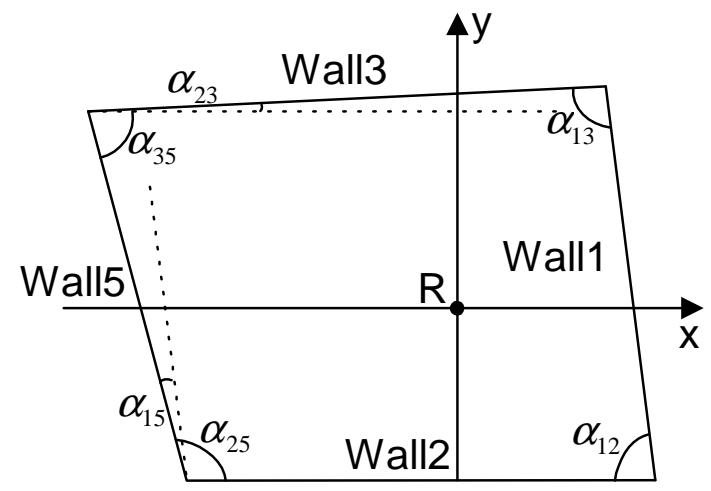

Fig. 19. Reconstructed map of the whole scenario

In this case, Wall3 is not 'flippable' relative to Wall2 on its own, without flipping Wall5 relative to Wall1, as they need to keep the same angle between them. Although, it is possible for both walls 3 and 5 to flip relative to walls 2 and 1, yet still keep the same angle between them, this happens only if $\alpha_{15}=\alpha_{23}$, which is very unlikely.

The reconstructed scenario in Fig. 19 can be optimised into a more accurate one using $\alpha_{12}, \alpha_{35}$ and the greater of $\alpha_{15}$ and $\alpha_{23}$. Though the part comprising walls 1 and 2 with the part comprising walls 3 and 5 are of high accuracy, the relative position between them is less accurate. Therefore, the optimised scenario is of low accuracy.

D. Scenarios With Three Obtuse and One Acute Inner Angle

Fig. 20 shows a single radio user operating in a closed fourwall environment with three obtuse and one acute inner angle. Again, for clearer explanations, obtuse and acute inner angles are exaggerated. The measured delay pattern consists of seven TOFs.

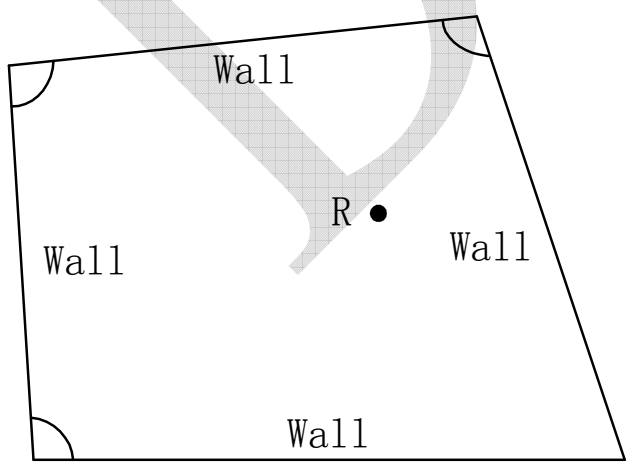

Fig. 20. A closed four-wall environment with 3 obtuse and 1 acute inner angle

By using the same method as described in section II.A, numbering walls according to the rank order of their corresponding TSs, it is found that: Wall1 and Wall4 are roughly parallel; Wall2 and Wall3 are roughly parallel; Wall1 and Wall3 are roughly perpendicular and adjacent. As Wall1 and Wall3 have acute angles with two other walls, while Wall2 and Wall4 have only one, either Wall1 or Wall3 can be used to define the coordinate system.

Using Wall3 to set up the coordinate system centred at the radio user with the path of the single reflection from Wall3 lying on the negative vertical axis, putting aside mirror-image ambiguity, Fig. 21 shows the relative position of Wall1, which is roughly perpendicular and adjacent to Wall3.

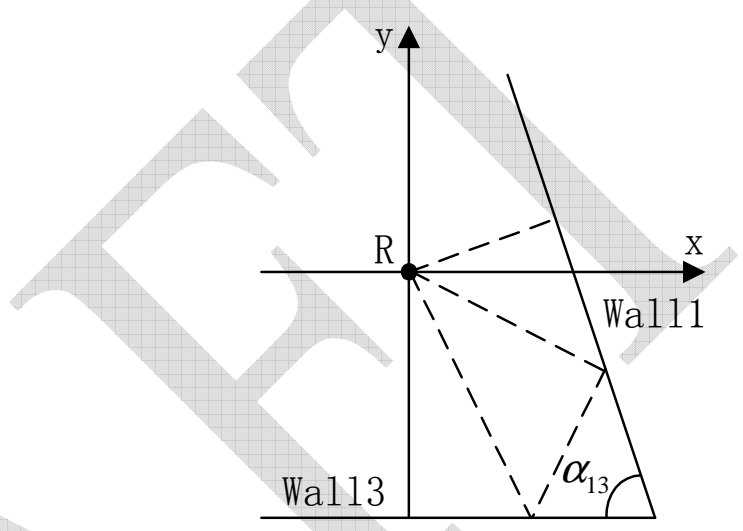

Fig. 21. Position of Wall1 in relation to Wall3 and the radio user when mirror-image ambiguity is not considered

Wall4 is roughly parallel to Wall1. Wall2 is roughly parallel to Wall3. Therefore, Wall4 is adjacent to Wall3 and Wall2. Wall2 is adjacent to Wall4 and Wall1. Based on the partial map in Fig. 21, a sketch of the whole scenario can be estimated as shown in Fig. 22.

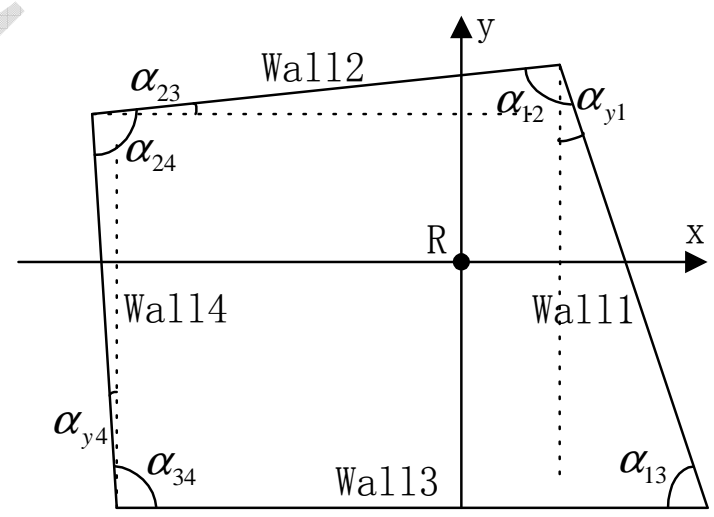

Fig. 22 Reconstructed map of the whole scenario

There could potentially be two flipping-wall ambiguities, i.e. Wall4 is 'flippable' relative to Wall1 and Wall2 is 'flippable' relative to Wall3. However, neither of these two walls is found to be 'flippable', which is proved as follows.

Apart from $\alpha_{13}$, all the other three inner angles between adjacent walls, i.e. $\alpha_{12}, \alpha_{24}$, and $\alpha_{34}$, are obtuse. Therefore,

$\alpha_{24}+\alpha_{34}>\pi$. 
In the coordinate system defined using Wall3 and the radio user (xRy in Fig. 22), the extension of Wall2 should intersect the negative horizontal axis, as shown in Fig. 22. Therefore, Wall2 is not 'flippable' relative to Wall3.

As $\alpha_{12}$ and $\alpha_{24}$ are obtuse, then

$\alpha_{12}+\alpha_{24}>\pi$

In the coordinate system defined using Wall1 and the user (x'Ry' in Fig. 23), the extension of Wall4 should intersect the positive horizontal axis, as shown in Fig. 23. Therefore, Wall4 is not 'flippable' relative to Wall1.

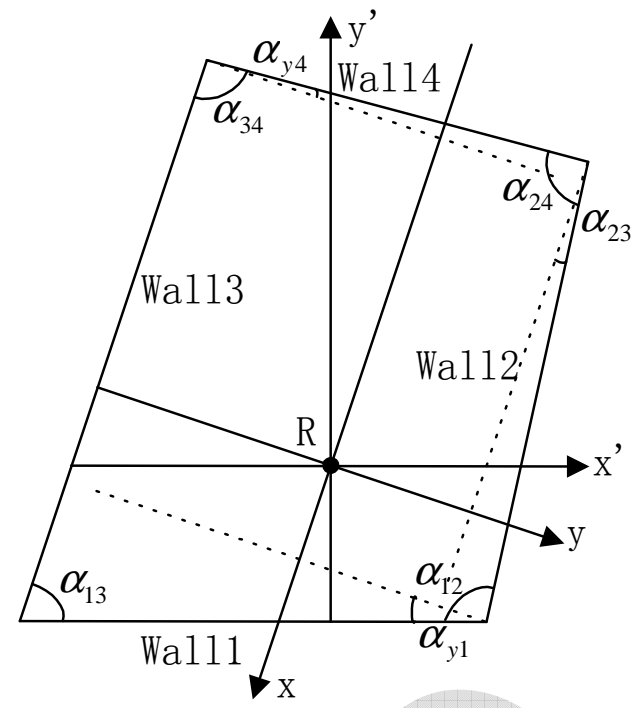

Fig. 23 Reconstructed map in the coordinate system defined by Wall1 and the radio (derived by rotating Fig. 22)

Based on this, we can conclude that, for two roughly parallel walls, flipping-wall ambiguity exists only when the side to which the extensions of these two walls will intersect is uncertain. In a four-wall environment, this requires the two walls both adjacent to the same pair of roughly parallel walls form two acute angles with one of them and two obtuse angles with the other. Therefore, flipping-wall ambiguity only exists in scenarios with two adjacent obtuse inner angles.

In Fig. 22, as there are only three acute angles available, i.e. $\alpha_{13}, \alpha_{14}$ and $\alpha_{23}$, and all of them are involved in the scenario reconstruction, there is no further optimisation to improve the accuracy. As the value of $\alpha_{14}$ and $\alpha_{23}$ are less accurate, though the part comprising walls 2 and 3 are of high accuracy, the whole scenario is of low accuracy.

\section{E. Mirror-image ambiguity}

So far, mirror-image ambiguity remains in the possible maps of the whole scenario. This ambiguity can be resolved if the radio user is detected to have moved twice in different directions. By repeating the mapping process at intervals that allow the radio user to make detectable movements, the path of the radio can be represented as a "track" in relation to the surroundings. If the radio has turned left, the correct "track" will also have turned left, whereas the "track" of the mirror image will have turned right, and vice versa. If the radio user knows which way he/she has turned, the ambiguity can be resolved. If the radios are carried by robots and their control circuits can tell which way they have turned within tens of milliseconds, the mirror-image ambiguity will be resolved within this short period of time. Human users may also be expected to know which way they have turned, given a longer period of time. If the system is used to track radio tags without this intelligence, some other means of measuring rotation may be needed, e.g. accelerometers.

\section{MORE RADIO USERS AND MORE COMPLEX ENVIRONMENTS}

Where there is more than one radio operating, each radio may derive a different sub-set of the structures in a complex environment "in view". They will therefore be able to exchange [19] partial maps to build up a more complete picture of a complex environment.

\section{CONCLUSION}

This paper describes a mapping and positioning technique for individual impulse radios to reconstruct a map representing the features of its surroundings. This technique has similarities to mono-static radar, but it does not require a directional antenna. By measuring the TOFs of pulses reflected by the surroundings using a single radio, this paper shows that, the map of typical four-wall indoor environments in relation to the radio can be successfully reconstructed. This technique can be extended to solve more complex scenarios.

\section{ACKNOWLEDGMENTS}

We would like to thank Prof. Stephen K. Barton, who is retired from the University of Manchester, for his valuable ideas, guidance and support of this research.

\section{REFERENCES}

[1] R. Want, A. Hopper, V. Falcao, and J. Gibbons, "The Active Badge Location System," ACM Trans. Information Systems, January 1992, vol. 10, pp. 91-102.

[2] A. Harter and A. Hopper, "A Distributed Location System for the Active Office," IEEE Network, January/February 1994, pp. 62-70.

[3] N.B. Priyantha, A. Chakraborty, and H. Balakrishnan, "The Cricket Location-Support System," in Proc. 6th Ann. Int'l Conf. Mobile Computing and Networking (Mobicom 00), ACM Press, New York, 2000, pp. 32-43.

[4] P. Bahl and V. Padmanabhan, "RADAR: An In-Building RF-Based User Location and Tracking System," in Proc. IEEE Infocom 2000, IEEE CS Press, Los Alamitos, California, 2000, pp. 775-784.

[5] J. Hightower, R. Want, and G. Borriello, "SpotON: An Indoor 3d Location Sensing Technology Based on RF Signal Strength," UW CSE 2000-02-02, Univ. Washington, Seattle, Feb. 2000.

[6] A.A.M. Saleh and R.A. Valenzuela, "A statistical model for indoor multipath propagation," IEEE Journal on Selected Areas in Communications, February 1987, pp. 123 - 137.

[7] P.F.M. Smulders, "Broadband Wireless LANs: A Feasibility Study," PhD thesis, Eindhoven University of Technology, Eindhoven, 1995.

[8] http://www.fcc.gov/oet/info/rules/part15/part15-91905.pdf

[9] S.Y. Seidel and T.S. Rappaport, "A ray tracing technique to predict path loss and delay spread inside buildings," in Proc. IEEE GLOBECOM'92 Conference, Orlando, USA, December 1992, pp. 649-653. 
[10] J. L. Richards, P. L. Jett, L. W. Fullerton, L. E. Larson and D. A. Rowe, "Precision Timing Cenerator Apparatus and Associated Methods," United States Patent, US6577691.

[11] G. Frenkel, "Geometric dilution of position (GDOP) in position determination through radio signals," in Proceedings of the IEEE, Vol. 61, Issue 4, April 1973, pp. 496 - 497.

[12] W. Guo, N.P. Filer and S.K. Barton, "A novel wireless mapping and positioning technique for impulse radio networks," in Proc. 18th triennial URSI International Symposium on Electromagnetic Theory, May 2004, Pisa, Italy, vol. II, pp. 712-714.

[13] W. Guo, N.P. Filer and S.K. Barton, "2D Indoor Mapping and Positioning Using an Impulse Radio Network," in Proc. the IEE Seminar on Ultra Wideband Communications Technologies and System Design, July 2004, London, UK, pp. 119-122.

[14] W. Guo, N.P. Filer and S.K. Barton, "2D Indoor Mapping and Location-sensing Using an Impulse Radio Network," in proc. the 2005 IEEE International Conference on Ultra-Wideband, September 2005
Zurich, Switzerland, pp. 296 - 301.

[15] W. Guo and N.P. Filer, "2.5D indoor mapping and location-sensing using an impulse radio network," in Proc. the IEE Seminar on Ultra Wideband Systems, Technologies and Applications, April 2006, London, UK, in press.

[16] M. Kmec, J. Sachs, P. Peyerl, P. Rauschenbach, R. Thoma and R. Zetik: "A novel Ultra-Wideband real-time MIMO Channel Sounder Architecture," XXVIIIth General Assembly of URSI, October 2005, New Delhi, India.

[17] www.meodat.com

[18] www.pulsers.net

[19] H. Cao, K. M. Nasr and S. K. Barton, "Echo Domain Multiple Access (EDMA), A new multiple access technique for impulse radio in a multipath environment," in Proc. 18th triennial URSI International Symposium on Electromagnetic Theory, May 2004, Pisa, Italy, vol. II, pp. 721-723. 